\title{
Best-worst scaling to assess the most important barriers and facilitators for the use of Health
} Technology Assessment in Austria

\begin{abstract}
Background: Although Health Technology Assessment (HTA) is increasingly used to support evidencebased decision-making in health care, several barriers and facilitators for the use of HTA have been identified. This best-worst scaling (BWS) study aims to assess the relative importance of selected barriers and facilitators of the uptake of HTA studies in Austria.

Methods: A BWS object case survey was conducted among 37 experts in Austria to assess the relative importance of HTA barriers and facilitators. Hierarchical Bayes estimation was applied, with the bestworst count analysis as sensitivity analysis. Subgroup analyses were also performed on professional role and HTA experience.

Results: The most important barriers were 'lack of transparency in the decision-making process', 'fragmentation', 'absence of appropriate incentives', 'no explicit framework for decision-making process', and 'insufficient legal support'. The most important facilitators were 'transparency in the decision-making process', 'availability of relevant HTA research for policy makers', 'availability of explicit framework for decision-making process', 'sufficient legal support', and 'appropriate incentives'.

Conclusion: This study suggests that HTA barriers and facilitators related to the context of decision makers, especially 'policy characteristics' and 'organization and resources' are the most important in Austria. A transparent and participatory decision-making process could improve the adoption of HTA evidence.
\end{abstract}

Keywords: Health Technology Assessment, barriers, facilitators, best-worst scaling, decision-making, stakeholders 


\section{Introduction}

In light of the resource scarcity and budget constraints that health systems are facing, healthcare-related decisions have to be made on the optimal and efficient allocation of resources [1]. Within this context, a trend towards evidence-based decision-making can be observed which reinforces the need to base resource allocation decisions on rational criteria, including (cost-)effectiveness, efficiency [2] and equity [3] considerations. The importance of the utilization of health-related research in policy-making has been widely recognized [4] and is increasingly based on Health Technology Assessment (HTA). HTA is a policyoriented form of research designed to examine the consequences of the introduction of health care technologies and interventions $[5,6,7]$. It aims to inform policy and clinical decision-making in health care [7] by assessing the medical, social, economic and ethical impact of the use of health technology [8].

Although HTA and its branch of economic evaluation is widely recognized as being an insightful tool to achieve health care efficiency, the impact on decision-making could still be limited [2]. The extent to which HTA information is utilized in decision-making varies across European countries, mainly due to the countries' different governmental and organizational structures in the health system $[9,10,11]$. In Austria, HTA research has been introduced in the 1990s. Since the establishment of the 'Ludwig Boltzmann Institute for Health Technology Assessment' (LBI-HTA) in 2006, HTA research has gained greater prominence by providing scientific support for decision-making in the Austrian health care sector [12, 13]. Nevertheless, Austria is a latecomer in implementing HTA evidence as decision-support tool compared to other countries $[13,14]$ and the use of HTA information by different national stakeholders still has potential for increase.

Since the gap between HTA research and policy uptake is often described as an issue $[9,15]$, several studies have investigated and identified multiple barriers of and incentives for the use of HTA information within the decision-making process internationally $[1,5,15,16,17]$. For instance, reported barriers include 'having no time/opportunity to use research evidence', 'difficulties in transferring budgets' or 'lack of credibility of HTA studies [5, 15], while reported facilitators include 'availability of and access to HTA findings', or 'explanation of the practical relevance of results' (e.g. actual cost savings) $[15,18]$. However, there is as paucity of research that assesses the most important barriers and facilitators. Only one Dutch best-worst scaling (BWS) study was identified suggesting that barriers and facilitators related to 'policy characteristics', and 'organization and resources' were particularly important [19]. Little is however 
known on how the Dutch BWS study results could be transferable to other countries. Moreover, in most of the published international studies, little attention has been given to the perspective of knowledge users of HTA evidence, i.e. policy makers $[5,16]$. Hence, it is of great relevance to also include knowledge users and investigate their perception of the practicability of HTA as well as their priorities $[5,15]$. For Austria, to optimize and increase the usage of HTA it is of great interest and relevance to elicit the most important barriers and facilitators to the adoption of HTA evidence by national stakeholders. Only when considering the various reasons for limited usage can a better management and utilization of scarce health care resources through improved uptake of HTA be realized [5].

The aim of this BWS study was therefore twofold: (1) to collect and analyse data on the relative importance of selected barriers and facilitators of the uptake of HTA studies in Austria, and (2) to explore differences in perception between different stakeholder groups. The study addresses the question which factors are considered most important and least important by relevant stakeholders, including Austrian policy makers, health professionals and HTA researchers. Such understanding will provide important insights for a better matching of HTA and user needs, and thereby potentially help strengthening the application of HTA. 


\section{Methods}

\subsection{Best-worst scaling}

The BWS technique, which is a type of conjoint analysis, is becoming increasingly popular to elicit preferences in health care $[20,21]$. BWS, compared to the traditional and more widely applied discrete choice experiment (DCE), presents an alternative preference elicitation method [22] that can resolve some of the limitations of the DCE technique [23]. For example, BWS is relatively simple to understand and thus supposed to reduce the cognitive burden for respondents and facilitates the evaluation of maximumdifference questions. BWS also overcomes the traditional 'pick one' task used in DCE [22] by eliciting additional information on both the most and least preferred option. Additionally, BWS possesses the ability to embrace a larger set of factors to determine preferences [23].

In this study, we used a BWS object case (case 1), also known as attribute case [22], to quantitatively assess the relative importance of an exhaustive list of nationally relevant barriers and facilitators to the adoption of HTA information. Respondents were asked to make judgements on the most and least important factor out of five elements of a choice set aiming for the analysis of preferences regarding a set of attributes/factors. This trade-off technique applied in the BWS survey constituted of two parts, one part addressing barriers and one part addressing facilitators.

\subsection{Identification of attributes}

The majority of attributes assessed in this study were based on a master list of 22 barriers and 19 facilitators which were identified in an international scoping review conducted for a comparable Dutch BWS study [19]. Further details on the scoping review can be found in Cheung et al. (2017) [19]. To take the specificity of the Austrian health care system and decision-making context into consideration, additional factors were identified through a scoping review of relevant peer-reviewed and grey literature on HTA in Austria conducted by CF and SM. This search in PubMed, Web of Science, google scholar and on websites of key stakeholders led to a total of 13 relevant studies. The additionally generated list of factors was discussed within the project group and further validated by three national experts (one policy maker and two HTA experts). This led to a final master list of 25 barriers and 21 facilitators for the BWS experiment. Appendix Table 1 lists these factors. As shown in appendix Table 2, these barriers and 
facilitators can be classified into three main categories and six sub-categories according to Brouselle and Lessard (2011) and Oliver et al. (2014) [2,15]. The three main categories relate to decision-maker, contextrelated and methodology-related factors. The six sub-categories (themes) include 'policy characteristics', 'organization and resources' 'contact and collaboration', 'research and researcher characteristics', 'policy makers characteristics' and 'other' [15].

\subsection{Survey participants}

A purposive sampling method [24] was applied to recruit relevant stakeholders in Austria, including policy makers, health professionals, HTA experts (i.e. research associates in the field of HTA and members of specific HTA research groups or institutes; PhD students were excluded), and the pharmaceutical industry, for participating in the survey. Important stakeholders with an interest were selected. The list of potential participants contained an equal sample size of experts from all stakeholder groups to potentially allow for an overall balanced assessment of HTA barriers and facilitators from different stakeholder perspectives.

The sample selection process was based on a systematic assessment of national HTA key players through several channels. Firstly, all HTA institutions listed in the Austrian HTA Guide [25] were included. Secondly, the list of potential participants was complemented with institutions and contacts provided by national experts of the Austrian health care system,

Additional stakeholders were also informed by institutions identified in a recent systematic literature review of published economic evaluations in Austria [26, 27]. Thirdly, relevant experts were selected based on the literature review conducted for the identification of Austriaspecific barriers and facilitators.

Participant contact information was collected between December 2016 and April 2017. The final list of participants consisted of a total of 96 experts.

2.4 The best-worst scaling survey

The Sawtooth Software's SSI ${ }^{\circledR}$ Web platform was used to design the BWS survey. Fractional, efficient designs were applied, characterized by: 1) orthogonality (factors were shown and paired an approximately equal number of times), 2) minimal overlap (minimizing the number of times each factor appears within 
the same set across the design), 3) positional balance (factors appear approximately an equal number of times in each position), 4) connectivity (factors are directly or indirectly linked), and 5) stability. Four different versions of the questionnaire were generated for each part of the BWS experiment, i.e. four versions for barriers and four versions for facilitators.

The online survey was designed via Qualtrics ${ }^{\circledR}$ and consisted of a self-administered questionnaire. The questionnaire included mainly closed-ended questions and two open-ended question providing participants the opportunity to give further comments on the survey content. Demographic and professional characteristics (gender, age, professional role, HTA experience) were assessed at the beginning. Each participant was asked to define their professional role by choosing one out of four different roles, including policy maker, health professional, research associate in the field of HTA or evidence-based research, and specific HTA research group or institute. To allow for a comparison with the findings of the recent Dutch BWS study [19], the categorization of professional roles was set-up similarly. Hence, stakeholder groups from the pharmaceutical industry were not indicated separately and due to the anonymous set-up of the survey, it remains unclear how many stakeholders from this subgroup eventually participated in the survey.

The relative importance of each individual factor was assessed per participant using 15 choice sets for barriers (the barriers questionnaire) and 13 choice sets for facilitators (the facilitators questionnaire), each composed of five factors from the master list. Participants were randomly assigned to one out of the four versions of the barriers questionnaire and one out of the four versions of the facilitators questionnaire while the order of the survey parts (barriers/facilitators) was also randomly allocated. At the end of the barriers and facilitators parts, participants were asked to rate the difficulty of completing the choice sets based on a Likert-scale (1=very easy, to 7=very difficult).

The BWS survey was sent out via Qualtrics ${ }^{\circledR}$ in May 2017. It was active for an initial period of two weeks and a weekly reminder was distributed out via Qualtrics ${ }^{\circledR}$. Respondents were assured anonymity throughout the survey procedure.

\subsection{Statistical analysis}


All completed surveys were included in the data analysis. In previous BWS object case studies, the Hierarchical Bayes (HB) estimation has been frequently used to analyse data $[21,28]$. Therefore, the HB estimation was used as the main analysis method in this study using the Sawtooth Software's SSI ${ }^{\circledR}$ Web platform. The calculation of the mean relative importance score (RIS) with its 95\% confidence interval, generated by the HB estimation, allowed ranking the factors from the most and least important. The higher the RIS score, the higher the relative importance of a factor (barrier or facilitator) was found.

In addition to the HB estimation, the best-worst count analysis was conducted as a secondary (sensitivity) analysis. The best-worst count analysis, which is a relatively simpler approach to analyse BWS object case data [21], is also increasingly applied in BWS object case studies [21]. Accordingly, we calculated scores based on the number of times an attribute was selected as the best and the worst across all questions included in the survey $[29,30]$. The number derived for the least important factor was subtracted from the count for the most important factor (total(best)-total(worst)) [31]. The obtained number was divided by the number of times the factor appeared in the survey, creating a scale $(-1$ to +1$)$ in which a high score indicates that the factor is more important to the respondent $[29,30]$.

The demographic and professional characteristics of the participants were described and used for further analyses. Subgroup analyses according to professional roles (policy makers vs. health professionals/HTA experts, including research associates in the field of HTA or evidence-based research, and members of HTA research groups or institutes) and HTA experience (based on the self-reported performance of an HTA study) were performed to assess whether the perception on the importance of factors differed by these stakeholder characteristics. With respect to the subgroup analysis on professional roles, the distinction between policy makers and health professionals/HTA experts assured an emphasis on the importance of the policy makers' role, which is considered a distinct and vital stakeholder group regarding the uptake of HTA evidence in Austria. Regarding HTA experience, health professionals and HTA experts were considered a homogenous group compared to policy makers, hence the latter were excluded in the subgroup analysis evaluating the influence of HTA experience on the perception of HTA barriers and facilitators. Statistical analyses were conducted in SPSS (version 23), including Chi-square tests and oneway analysis of variance (ANOVA). A p-value $<0.05$ was considered statistically significant. 


\section{Results}

\subsection{Participant characteristics}

In total, 53 participants started the survey. Out of these, 37 participants (69.8\%) completed the survey and were included in the analysis, resulting into an overall response rate of $38.5 \%$ (37/96). Seven incomplete responses of those who started the survey contained information on the demographic and professional characteristics; no significant differences were found between these participants $(n=7)$ and those who completed the survey $(n=37)$.

Seventeen survey participants were male (45.9\%) and 20 participants were female (54.1\%). The age of the respondents ranged from $20-29$ years $(5.4 \%, n=2), 30-39(24.3 \%, n=9), 40-49(32.4 \%, n=12), 50-59(24.3 \%$, $n=9)$ to $60-69$ years $(13.5 \%, n=5)$. There were six policy makers (16.2\%), five health professionals $(13.5 \%)$, 21 research associates (56.8\%) and five members of HTA research groups or institutes (13.5\%). In terms of HTA experience, almost three-quarter of the participants ( $n=27 ; 73.0 \%$ ) self-reported having conducted or contributed to an HTA study at least once during their professional career, whereas ten participants (27.0\%) reported that they had not previously conducted an HTA study.

Participants rated both BWS experiment parts as medially difficult on a 7-point Likert scale (barriers: mean=4.02, SD=1.55; facilitators: mean=4.08, SD=1.66). Six comments were provided in the open-ended questions fields, either related to the difficulty of completing the choice tasks, pointing out the lacking availability of HTA evidence or emphasizing the importance of a regulatory framework for HTA in Austria.

The overall fit statistic (barriers $=0.45$; facilitators $=0.48$ ) of the responses was considered good. All respondents had an overall fit statistic higher than 0.25 and were thus all included in the analysis [32].

\subsection{Relative importance of HTA barriers}

Figure 1 illustrates the RIS of the barriers and points at a visual cut-off point (RIS=6.50) that can be considered as a threshold to differentiate the most important factors from the remaining attributes in this study. 
The five most important barriers (RIS>6.50) to the uptake of HTA in Austria $(n=37)$ were 'lack of transparency in the decision-making process', 'fragmentation' (difficulties in moving resources/budgets from one sector to another), 'absence of appropriate incentives for implementation of cost-effective interventions', 'no explicit framework for decision-making process' (that uses HTA evidence), and 'insufficient legal support' (lack of legal or legislative support to the use of HTA).

The best-worst count analysis, which was conducted as a sensitivity analysis, yielded similar results regarding the ranking order of the most important barriers (see Appendix Table 3).

Figure 1. Ranked barriers of the uptake of HTA $(n=37)$.

The RIS in boxes indicate the most important barriers (RIS>6.50).

Based on a categorization of all selected barriers from the list of factors included in this study ( $n=25)$, context-related factors $(n=12)$, referring to the sub-categories 'policy characteristics' and 'organization and resources' [15] comprised overall the highest number of factors, followed by methodology-related $(n=7)$ and decision-maker related $(n=6)$ factors (see Figure 1 and Appendix Table 2). Following the classification of the five most important barriers revealed that 'context-related factors' [2] were the most dominant themes. The least important barriers determined in this study were methodology-related factors that refer to the sub-category 'research and researcher characteristics' [15], and include barriers such as 'no guidelines' (absence of adequate/reliable HTA guidelines) and 'inadequate presentation format' (HTA reports overly long, too theoretical or abounding in technical jargon).

The subgroup analysis demonstrated two statistically significant $(p<0.05)$ differences between policy makers and health professionals/HTA experts. As shown in Figure 2, the factor 'lack of qualified human resources to conduct or understand relevant HTA research within the policy organization' was considered more important by policy makers. The factor 'no explicit framework for decision-making process' was perceived as more important by health professionals/HTA experts. Although not statistically significant, additional differences in perception are indicated in Figure 2. It seems that 'absence of appropriate incentives for implementation of cost-effective interventions' and 'insufficient legal support' (lack of legal support or legislative support to the use of HTA) were more important for health professionals/HTAexperts. In contrast, policy makers emphasized that 'lack of transparency of HTA research findings' (the process as to how presented research findings emerged is not clear) may be an important barrier to the 
use of HTA evidence as well as encountering 'insufficient quality of HTA research findings' (quality not according scientific requirements).

Figure 2. HTA barriers by policy makers $(n=6)$ and health professionals/HTA experts $(n=31)$.

* and boxes indicate a statistically significant factor, $p<0.05$.

The other subgroup analysis revealed two statistically significant $(p<0.05)$ differences between participants who reported that they had already conducted an HTA study in their professional career and participants who did not (results not shown, $n=31$ ). 'Lack of longstanding relation between professionals/policy makers and researchers' (mean $=3.18, S D=2.78$ vs. mean $=5.70, S D=3.04, F=4.29$ ) and 'no access to relevant HTA research (poor dissemination) for policy makers' (mean=1.32, SD=1.00 vs. mean=2.96, $\mathrm{SD}=3.09, \mathrm{~F}=5.32$ ) were more important for health professionals and HTA experts without applied experience in conducting an HTA study.

\subsection{Relative importance of HTA facilitators}

Figure 3 presents the RIS of the facilitators. The factors 'transparency in the decision-making process', 'availability of relevant HTA research for policy makers', 'availability of explicit framework for decisionmaking process' (that uses HTA evidence), 'sufficient legal support' (legal or legislative support to the use of HTA), and 'appropriate incentives for implementation of cost-effective interventions' were considered as being most important for Austrian experts (RIS>6.50).

Similarly to the findings of HTA barriers, the best-worst count analysis resulted in a comparable ranking order of the most important facilitators (see Appendix Table 3).

Figure 3. Ranked facilitators of the uptake of HTA ( $n=37)$.

The RIS in boxes indicate the most important facilitators (RIS>6.50).

Classifying all facilitators listed in this study shows that overall, context-related factors $(n=10)$ were highest in number, followed by decision-maker related $(n=6)$ and methodology-related factors $(n=5)$ (see Figure 3 and Appendix Table 2). Categorizing the five most important facilitators into categories and subcategories revealed that here also 'context-related factors' [2] were the most important. The least 
important HTA facilitators assessed in this study were the following: 'appropriate HTA topic-finding process' (context-related factor) and 'availability of guidelines' (the availability of adequate/reliable HTA guidelines) (methodology-related factor).

The subgroup analysis revealed three statistically significant $(p<0.05)$ differences between policy makers and health professionals/HTA experts (Figure 4). 'Sufficient awareness within the organization to the relevance of HTA' and 'appropriate HTA topic-finding process' were more important for policy makers than for health professionals/HTA experts while 'appropriate incentives for implementation of costeffective interventions' were perceived more relevant for health professionals/HTA experts than for policy makers. Figure 4 shows all (also statistically non-significant) differences in perception regarding facilitators to the use of HTA evidence in decision-making. Policy makers found 'sufficient support by stakeholders' (policy maker's perception of insufficient support by end-users) as an important facilitator. Contrary, health professionals/HTA experts perceived that 'sufficient financial resources to conduct relevant HTA research' presents an important facilitator to the adoption of HTA evidence.

No statistically significant difference was found according to HTA experience.

Figure 4. HTA facilitators by policy makers $(n=6)$ and health professionals/HTA experts $(n=31)$.

* and boxes indicate a statistically significant factor, $p<0.05$. 


\section{Discussion}

This study identified the relative importance of barriers and facilitators to the uptake of HTA evidence in Austria as well as the differences in perception between policy makers, and health professionals/HTA experts in this respect. Our findings indicate that especially the Austria-specific HTA barriers and facilitators had a greater relative importance compared to the internationally validated HTA factors.

Many of the identified most important HTA barriers and facilitators were opposites. For instance, 'appropriate incentives for implementation of cost-effective interventions' was perceived as one of the most important facilitators, while 'absence of appropriate incentives for implementation of cost-effective interventions' was an important barrier. Classifying the five most important barriers and facilitators into categories [2] revealed that 'context-related factors' were the most important, which refer to the decision maker's context including organizational, political, budgetary and social factors [2]. Similarly to our findings, 'context-related factors' were identified as being the most important barriers and facilitators in a recent Dutch BWS study [19]. Therein, 'policy characteristics' (e.g. 'explicit framework for decisionmaking process that uses HTA evidence') and 'organization and resources' (e.g. 'availability of relevant HTA research for policy makers') were acknowledged to be the most significant sub-categories [19]. In accordance with our findings, Oliver et al. (2014) [15] suggest that factors relating to 'organization and resources' (e.g. 'availability of and access to research/improved dissemination') are most frequently reported barriers and facilitators for the use of HTA evidence. Especially, the high importance of the 'availability of relevant HTA research for policy maker' found in this study points out that (public) investment to help produce relevant HTA research in Austria, particularly matching the user needs of policy makers needs to be encouraged. This indication was supported by the comment made by a survey participant who emphasized the currently lacking availability of HTA evidence that is useful not only for the seller of a health technology, but for the decision maker. Contradictory to our findings, Oliver et al. (2014) [15] concluded that 'policy characteristics' such as legal support to the use of HTA and explicit framework for the use of HTA evidence were scarcely reported as factors affecting the uptake of HTA information internationally. In contrast, the least important factors determined in this study were related to 'research and researcher characteristics' [15], including i.e. absence/availability of HTA guidelines and the inadequate/clear presentation format of research findings. 
'(Lack of) transparency in the decision-making process' within the health care sector was found to be the most important barrier and facilitator to the adoption of HTA evidence in Austria. This factor was scarcely reported internationally and seems to be Austria-specific. This finding supports the rationale behind the assessment of country-specific factors to take into account the national health care and HTA landscape as well as the specific features of governmental, legal and organisational structures in the health care decision-making process. In addition, the high importance of both 'transparency in the decision-making process' and 'explicit framework for decision-making process' revealed, in line with the cross-sectional survey by Mochón et al. (2017) [11], that a transparent and comprehensive HTA system is an important prerequisite for the successful adoption of HTA evidence. Our study suggests that based on the perception of the surveyed stakeholders, such a system does not exist in Austria. Reasons for this may be, on the one hand, the unavailability or insufficient quality of local HTA input data, and on the other hand, the fragile structure/framework in HTA decision-making [11]. The former point was also supported by a survey participant who emphasized the availability of high quality input data as a crucial prerequisite to produce high quality HTA evidence ("garbage in, garbage out"). Mochón et al. (2017) [11] advise that improving transparency in the HTA decision-making process needs to be encouraged, while considering the necessity of an equilibrium between transparency and confidentiality. In this respect, our finding is in accordance with an earlier Austrian report by Schumacher and Zechmeister (2011) [33] and emphasizes that improved transparency in the decision-making processes is urgently needed. On the other hand, the report by Schumacher and Zechmeister additionally indicates that some evidence for a standardized inclusion of HTA results in the decision-making processes has been existing in Austria, linked "with tendencies to [enhance] transparency" [33]. Our subgroup analysis demonstrated that the relative importance of having 'no explicit framework for decision-making process' (that uses HTA evidence) was considered high dominantly by health professionals/HTA experts, implying that policy makers may have failed to consider this issue sufficiently.

'Fragmentation', capturing the difficulties in moving resources/budgets from one sector to another (i.e. fragmentation of responsibilities and competencies for HTA) represented the second most important rated barrier to the adoption of HTA. Indeed, the Austrian health care system is characterized by decentral decision and funding structures and a strong sectoral focus of relevant funding bodies [14]. The results of this BWS study confirm that the structure currently in place for implementing HTA evidence might need improvement, potentially also through strengthening the relevant legal framework. 
'(In)sufficient legal support' ((no) legal or legislative support to the use of HTA) was among the most important HTA barriers and facilitators in our study. This indicates, in line with Chinitz (2004) [9] and Mochón et al. (2017) [11] that the governance and organization of HTA systems are essential factors for influencing the uptake of HTA. Internationally, countries which reported having legislation establishing the use of HTA as mandatory, showed greater support of HTA evidence in the decision-making process [11]. Since health care in Austria is considered as the responsibility of public policy [13], a stronger centralization of HTA could potentially increase the impact on HTA adoption and its usage at both national clinical and policy level [9]. Consequently, legislative support for using HTA could possibly enhance the extent to which HTA evidence is adopted in Austria. This was also pointed out by a study participant, confirming that as long as there was no regulatory framework requiring federal states or health insurance funds to consider HTA evidence in their decision-making process, also little funding would be invested in the production of HTA reports by public authorities. However, at this point, it is worth mentioning that some legislative support of HTA usage can be observed with Article 15a of the Austrian Federal Constitution Law $(\S 17(4), 2013)$ calling for a "uniform framework of cost-benefit analyses and evidencebasing (HTA) for diagnosis and treatment methods (including health promotion, screening and vaccination programs)" [34].

Further subgroup analyses revealed several differences in the perception of stakeholders based on their professional roles and HTA experience. For example, 'lack of qualified human resources' (to conduct or understand relevant HTA research) was perceived as more relevant by policy makers. This finding stresses that profound understanding of HTA studies is an important prerequisite for adopting the derived evidence-based recommendation. Closely related to this, 'sufficient awareness' (within the organization to support the relevance of HTA) needs to be promoted. Moreover, an 'appropriate HTA topic-finding process', i.e. the existence of a participatory topic-finding system, was found to be more important for policy makers and points at a perceived gap in this respect. This means that the existence of a participatory topic-finding system, which allows regular and problem-relevant prioritization of HTA topics, is less essential for health professionals/HTA experts. Accordingly, it appears that the producers of HTA evidence may need to enhance their efforts to involve policy makers in their topic-finding process. Since 'appropriate incentives for implementation of cost-effective interventions' was more important for health professionals/HTA experts, reinforcing the factor 'contact and collaboration' (e.g. by improving longstanding relations between professionals/policy makers and researchers) could potentially lead to a better matching of user needs and an application of HTA [15]. 


\subsection{Strengths and limitations}

Previous studies have investigated multiple barriers and facilitators for the use of Health Technology Assessment evidence within the decision-making process $[1,5,15,16,17]$, however, few studies have yet quantitatively assessed the most important barriers and facilitators and involved decision-makers in addition to HTA experts $[19,33]$. To our knowledge, this is the first study assessing the relative importance of barriers and facilitators to the use of HTA in Austria, i.e. a country which can be considered a latecomer in implementing HTA evidence as decision-support tool compared to other countries $[13,14]$. In this study, both the initial international master list of factors and the additional list of Austria-specific factors were based on a literature review and validated by (Austrian) policy makers and HTA experts. The review of peer-reviewed and grey literature to identify Austria-specific factors constitutes important strength of this study. Accordingly, this allowed for a comprehensive assessment of HTA barriers and facilitators taking into account the country-specific institutional background, which should also be considered in future research. The factor list assessed substantially extends the six HTA impact indicators included in the earlier Austrian report by Schumacher and Zechmeister [33]. In our study, participants were selected from all relevant HTA institutions and an equal sample size from each stakeholder institution was included to potentially allow for an overall balanced assessment of HTA barriers and facilitators from different stakeholder perspectives. Moreover, an experienced sample partook in this survey with most participants ( $n=27,73 \%$ ) reported having conducted or contributed to conducting an HTA study during their professional career. Although this information is based on self-reported data and needs to be interpreted with caution, it suggests that this BWS study captures an insider perspective within the Austrian HTA field. Finally, two statistical methods were applied in this BWS study, yielding similar results and hence confirming the robustness of our findings.

There are, however, a number of limitations worth mentioning. Firstly, as some of the factors used in the BWS study were tailored to the Austrian institutional background, the findings of this study may not necessarily be generalizable to other countries, especially countries showing differences in characteristics of their HTA decision-making process (e.g. UK) [9]. At the same time, it is unclear if similar barriers and facilitators would be identified in countries with a similar tradition in HTA uptake as in Austria. Future research should look into this. Secondly, the overall results of the relative importance of HTA barriers (Figure 1) and facilitators (Figure 3) may be distorted due to the overrepresentation (e.g. HTA researchers) 
and underrepresentation (e.g. policy makers) of different stakeholder groups participating in the survey. Findings were therefore also presented by stakeholder groups (Figure 2, Figure 4). Thirdly, one may argue that the different study participants were influenced in their perceptions on the relative importance of different factors by underlying administrative variables such as if HTA reports are mandatory for a formal decision on pricing and reimbursement of a technology. At the same time, there is no such mandatory requirement to use HTA evidence in decision-making in Austria $[35,36]$ and albeit being a 'fourth hurdle' for innovative pharmaceuticals [37], economic evaluations also play a minor role in practice [38]. Expectedly, however, e.g. survey participants from the pharmaceutical industry might be influenced in their responses by this context, while the extent of their survey participation remains unclear. Consequently, formal assessment of this hypothesis is not possible and would have to be addressed by future research. Fourthly, study participants may not have been familiar with the BWS set-up as it represents a relatively novel method. This may have led to some respondents opting out the survey and hence a non-response bias. Although it is argued that a decreased cognitive burden is placed on participants facing a BWS choice format, the available evidence is not yet conclusive [39]. Finally, while this BWS study was a vital first step towards systematically identifying the most relevant barriers and facilitators for the uptake of HTA in Austria, further in-depth insights would be needed to understand how current processes could be improved. To this end, e.g. qualitative research including expert interviews on the identified main factors could be conducted to gain a more differentiated understanding of the underlying mechanisms.

\subsection{Policy making implication and recommendations}

Eliciting preferences in healthcare and adopting this knowledge to improve the use of HTA evidence in decision-making processes are important but have been scarcely performed. In this context, BWS showed a rapid development in health applications to elicit powerful stakeholder (public) preferences $[21,39,40]$. Regarding the adoption of HTA information, more in-depth insights into barriers and facilitators are needed to enhance the use of HTA in decision-making. Thus, identifying firstly the most important barriers and facilitators as done in this study seems a pre-requisite to the enhancement of both evidence-based decision-making and policy making. Once investigated, new methods need to be considered to improve policy priorities and decision-making support for HTA uptake to overcome the barriers described [15]. For instance, providing the required impulse at national level to further establish an explicit framework or even legal support for including HTA in the decision-making process on different levels based on fully 
transparent and formalized criteria seems to be an important step for Austria. The basis for Austria to improve their formal HTA process could be a cross-country comparison and learning from the experiences of other countries. Such insights could improve the application of HTA evidence and strengthen the implementation of cost-effective interventions in light of resource scarcity and budget constraints. 


\section{Conclusion}

This study aimed to quantitatively analyse the relative importance of barriers and facilitators regarding the use of HTA evidence in Austria. HTA barriers and facilitators related to the context of decision makers, including 'policy characteristics' and 'organization and resources' were found to be particularly important. Considering the importance of 'policy characteristics' factors, a higher emphasis should be set at the national level towards establishing an explicit framework or even legal support for including HTA evidence in health care decision-making in Austria. Hereby, the focus should lie on bridging the gap between HTA research and policy uptake on the one hand, and on ensuring a more transparent and participatory decision-making process on the other hand. 


\section{Key issues}

- Previous studies have investigated multiple barriers and facilitators for the use of Health Technology Assessment (HTA) evidence within the decision-making process internationally, however, few studies have yet quantitatively assessed the most important barriers and facilitators and involved decision-makers in addition to HTA experts.

- Results from this best-worst scaling (BWS) online survey indicate that HTA barriers and facilitators related to the context of decision-makers, including 'policy characteristics' (e.g. (lack of) transparency in the decision-making process) and 'organization and resources' (e.g. (absence of) appropriate incentives for implementation of cost-effective interventions) are particularly important in Austria.

- Subgroup analyses (policy makers vs. health professionals/HTA experts) show that the relative importance of having 'no explicit framework for decision-making process' as a barrier was more important for health professionals/HTA experts than for policy makers. Accordingly, policy makers may have failed to recognize this issue sufficiently and to provide an explicit framework to include HTA evidence in decision-making.

- A higher emphasis should be set towards establishing an explicit (legal) regulatory framework for including HTA evidence in health care decision-making on different levels, focusing on bridging the gap between HTA research and policy uptake on the one hand, and ensuring a more transparent and participatory decision-making process on the other hand. 


\section{References}

Papers of special note have been highlighted as either of interest $\left({ }^{*}\right)$ or of considerable interest $\left({ }^{* *}\right)$ to readers.

1. Eddama O, Coast J. A systematic review of the use of economic evaluation in local decisionmaking. Health Policy. 2008;86(2):129-141.

2. Brousselle A, Lessard C. Economic evaluation to inform health care decision-making: promise, pitfalls and a proposal for an alternative path. Social Science \& Medicine. 2011;72(6):832-839.

3. Panteli D, Kreis J, Busse R. Considering Equity In Health Technology Assessment: An Exploratory Analysis of Agency Practices. International Journal of Technology Assessment in Health Care. 2015;31(5):314-323.

4. Hanney SR, Gonzalez-Block MA, Buxton MJ, et al. The utilisation of health research in policymaking: concepts, examples and methods of assessment. Health Research Policy and Systems. 2003;1(1):2.

5. Hivon M, Lehoux P, Denis J-L, et al. Use of health technology assessment in decision making: Coresponsibility of users and producers? International Journal of Technology Assessment in Health Care. 2005;21(02):268-275.

*The limitations to the use of HTA are investigated based on semi-structured interviews.

6. Eddy D. Health Technology Assessment and Evidence-Based Medicine: What Are We Talking About? Value in Health. 2009;12(s2):S6-S7.

7. World Health Organization. Health Technology Assessment 2017; [cited 2017 May 23]. Available from: http://www.who.int/health-technology-assessment/about/en/

8. European network for Health Technology Assessment. What is Health Technology Assessment (HTA) n.d.; [cited May 22, 2017]. Available from: http://www.eunethta.eu/about-us/faq $\underline{\mathrm{t} 287 \mathrm{n} 73}$

9. Chinitz D. Health technology assessment in four countries: response from political science. International Journal of Technology Assessment in Health Care. 2004;20(1):55-60.

*This study assesses the importance of institutional structures that produce HTA and assesses their influence on health policy decision-making.

10. Nicod E, Kanavos P. Commonalities and differences in HTA outcomes: a comparative analysis of five countries and implications for coverage decisions. Health Policy. 2012;108(2):167-177.

11. García-Mochón L, Balbino JE, de Labry Lima AO, et al. HTA and decision-making processes in Central, Eastern and South Eastern Europe: Results from a survey. Health Policy. 2017.

*A cross-sectional study among CESEE countries investigating differences in HTA decisionmaking processes.

12. Schumacher I, Zechmeister I. Auswirkungen der HTA-Forschung auf das Gesundheitswesen in Österreich. Teil Methodenübersicht - Update. Vienna: Ludwig Boltzmann Institut für Health Technology Assessment. 2010.

13. Wild C. Austria: History of health technology assessment during the past 20 years. International Journal of Technology Assessment in Health Care. 2009;25(S1):74-81.

14. Antony K, Fröschl B, Rosian-Shikuta I, et al. Health Technology Assessment - Einsatz und Bedarf in Österreich und Implikationen für die Verankerung in Österreich. Vienna: Gesundheit Österreich GMBH. 2009.

15. Oliver K, Innvar S, Lorenc T, et al. A systematic review of barriers to and facilitators of the use of evidence by policymakers. BMC Health Services Research. 2014;14(1):2.

**A systematic review that identified the most frequently reported barriers and facilitators influencing the use of HTA evidence. 
16. Hoffmann C, von der Schulenburg J-MG. The influence of economic evaluation studies on decision making: A European survey. Health Policy. 2000;52(3):179-192.

17. Drummond $\mathrm{M}$, Weatherly $\mathrm{H}$. Implementing the findings of health technology assessments. International Journal of Technology Assessment in Health Care. 2000;16(01):1-12.

18. Van Velden ME, Severens JL, Novak A. Economic evaluations of healthcare programmes and decision making. Pharmacoeconomics. 2005;23(11):1075-1082.

19. Cheung KL, Evers S, Vries H, et al. Most important barriers and facilitators regarding the use of Health Technology Assessment. International Journal of Technology Assessment in Health Care. 2017;33(2):1-9.

**A recent Dutch best-worst scaling study assessing the most important HTA barriers and facilitators in The Netherlands.

20. Bridges JF, Hauber AB, Marshall D, et al. Conjoint analysis applications in health-a checklist: a report of the ISPOR Good Research Practices for Conjoint Analysis Task Force. Value in Health. 2011;14(4):403-413.

21. Cheung KL, Wijnen BF, Hollin IL, et al. Using Best-Worst Scaling to Investigate Preferences in Health Care. PharmacoEconomics. 2016;34(12):1195-1209.

22. Potoglou D, Burge P, Flynn T, et al. Best-worst scaling vs. discrete choice experiments: an empirical comparison using social care data. Social Science \& Medicine. 2011;72(10):1717-1727.

23. Flynn TN, Louviere JJ, Peters TJ, et al. Best-worst scaling: what it can do for health care research and how to do it. Journal of Health Economics. 2007;26(1):171-189.

24. Guarte JM, Barrios EB. Estimation under purposive sampling. Communications in StatisticsSimulation and Computation ${ }^{\circledR}$. 2006;35(2):277-284.

25. Austrian Public Health Institute; [cited May 30, 2017]. HTA-Guide. Available from: http://htaguide.bigg.at/?q=en/node/60

26. Mayer S, Kiss N, Laszewska A, et al. Costing evidence for health care decision-making in Austria: A systematic review. PLoS One. 2017;12(8):e0183116.

27. Mayer S, Kiss N, Laszewska A, et al. Health Economic Costing Methods and Reporting in Austria. Value in Health. 2016;19(7):A363.

28. Mühlbacher AC, Kaczynski A, Zweifel P, et al. Experimental measurement of preferences in health and healthcare using best-worst scaling: an overview. Health Economics Review. 2016;6(1):2.

29. Lee JA, Soutar GN, Louviere J. Measuring values using best-worst scaling: The LOV example. Psychology \& Marketing. 2007;24(12):1043-1058.

30. Sawtooth Software. Counting Analysis; [cited June 5, 2017]. Available from: https://www.sawtoothsoftware.com/help/lighthousestudio/manual/hid web maxdiff counting.html

31. Mühlbacher AC, Kaczynski A, Zweifel P. Experimentelle Präferenzmessung im Gesundheitswesen mit Hilfe von Best-Worst Scaling (BWS). PharmacoEconomics German Research Articles. 2013;11(2):101-117.

32. Sawtooth Software. Fit Statistic and Identifying Random Responders; [cited June 5, 2017]. Available from: https://www.sawtoothsoftware.com/help/lighthousestudio/manual/hid web maxdiff badrespondents.html

33. Schumacher I, Zechmeister I. Auswirkungen der HTA-Forschung auf das Gesundheitswesen in Österreich. Teil 2. Ergebnisse der empirischen Erhebung. Projektbericht. Nr. 37b . Vienna: Ludwig Boltzmann Institut für Health Technology Assessment. 2011.

**A highly relevant project report based on a mixed method assessment of HTA impact categories among relevant stakeholders in Austria. 
34. Federal Chancellery. 15a B-VG Zielsteuerung-Gesundheit; [cited June 19, 2017]. Available from: https://www.ris.bka.gv.at/GeltendeFassung.wxe?Abfrage=Bundesnormen\&Gesetzesnummer=2 $\underline{0008611}$

35. Zechmeister I, Schumacher I. The impact of health technology assessment reports on decision making in Austria. International Journal of Technology Assessment in Health Care. 2012;28(1):77-84.

36. Schumacher I, Zechmeister I. Assessing the impact of health technology assessment on the Austrian healthcare system. International Journal of Technology Assessment in Health Care. 2013;29(1):84-91.

37. Krammer H. Pharmakoökonomische Analysen - Chance oder 4. Hürde für innovative Arzneimittel. Wiener Medizinische Wochenschrift. 2006;156(23-24):606-611.

38. Walter E, Zehetmayr S. Guidelines zur gesundheitsökonomischen Evaluation Konsenspapier. Wiener Medizinische Wochenschrift. 2006;156(23-24):628-632.

39. Severin F, Schmidtke J, Mühlbacher A, et al. Eliciting preferences for priority setting in genetic testing: a pilot study comparing best-worst scaling and discrete-choice experiments. European Journal of Human Genetics. 2013;21(11):1202-1208.

40. Prosser LA. Statistical Methods for the Analysis of Discrete-Choice Experiments: A Report of the ISPOR Conjoint Analysis Good Research Practices Task Force. Value in Health. 2016;19(4):298299. 